
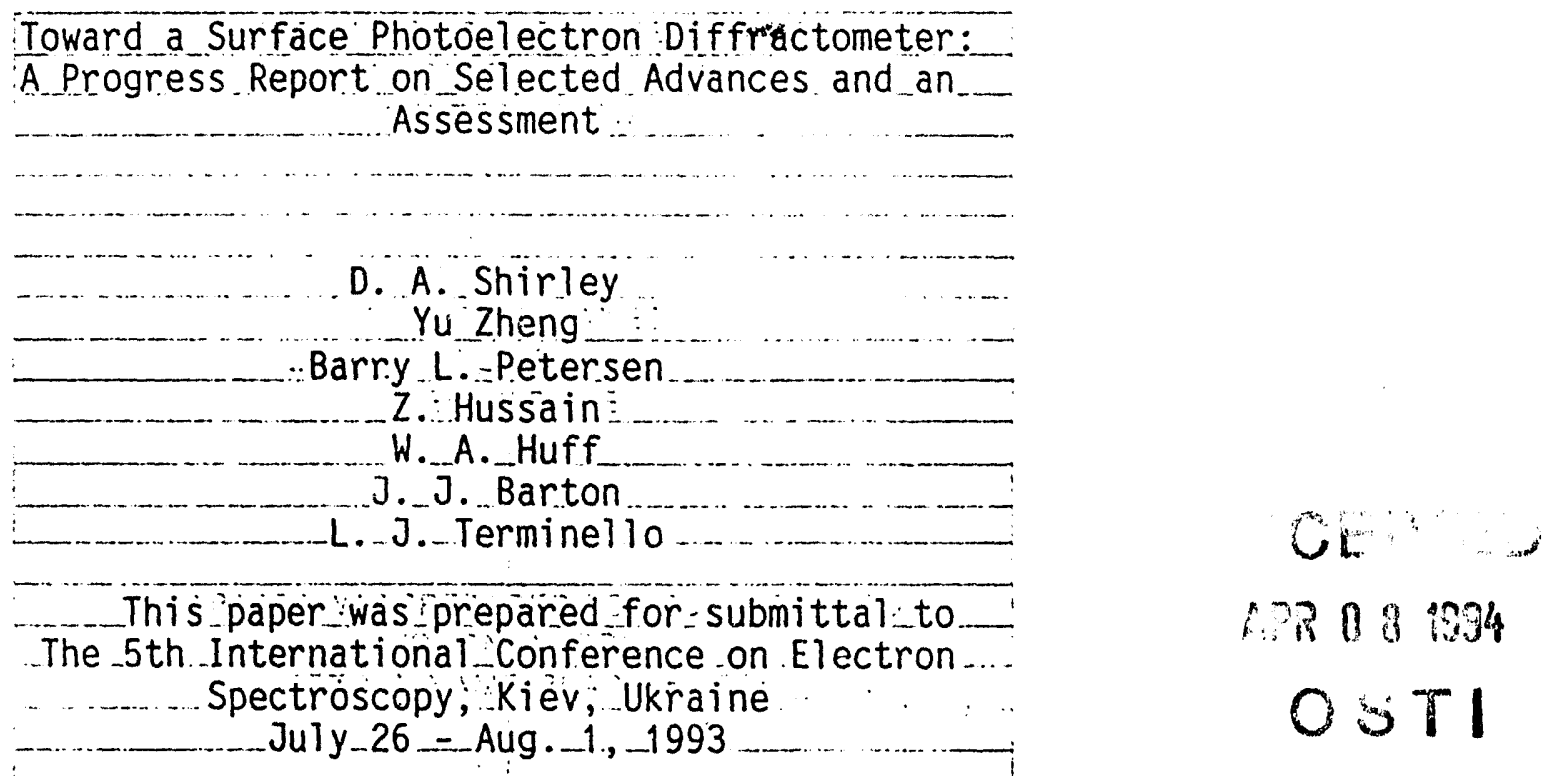

October $6 ; 1993$

\title{
3
}

- This is a preprint of a paper intended for publication in a journal or proceedings. Since changes may be made before publication, this preprint is made available with the understanding that it will not be cited or reproduced without the permission of the author ASSIFICATION CATEGOFY STATEIVIE:T
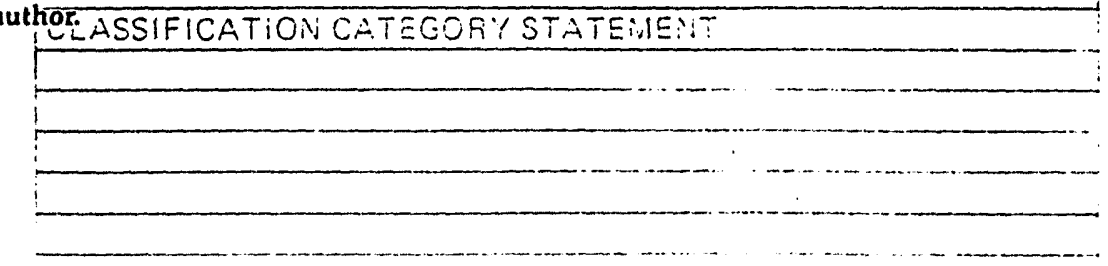

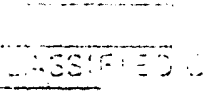

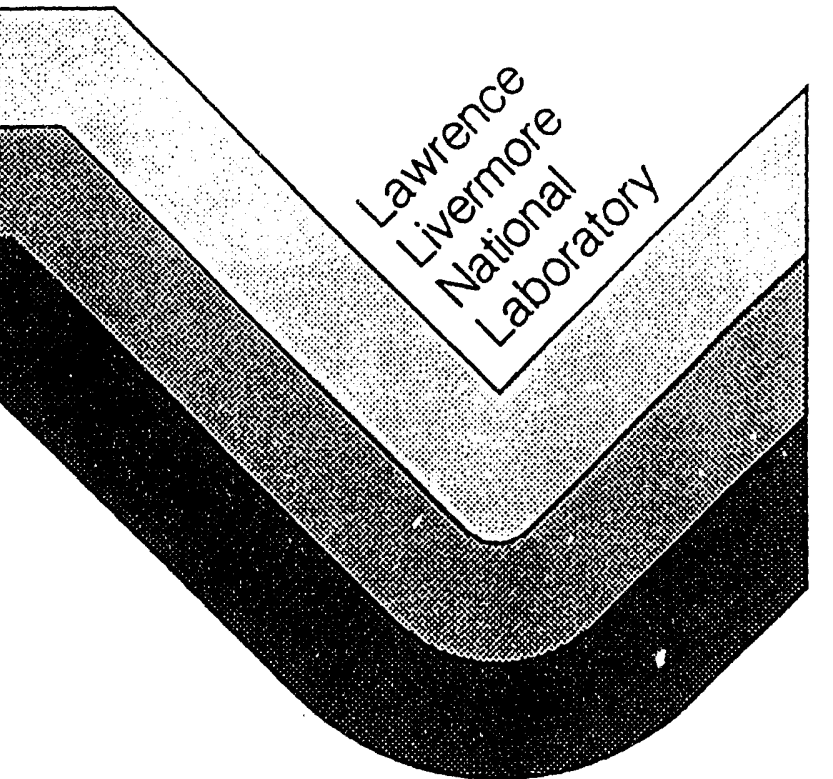

asira 
This document was prepared as an account of work sponsored by an agency of the United States Government. Neither the United States Government nor the University of California nor any of their employees, makes any warranty, express or implied, or assumes any legal liability or responsibility for the accuracy, completeness, or usefulness of anj information, apparatus, product, or process disclosed, or represents that its use would not infringe privately owned rights. Reference herein to any specific commercial products, process, or service by trade name, trademark, manufacturer, or otherwise, does not necessarily constitute or imply its endorsement, recommendation. or favoring by the United States Government or the University of Califurnia. The views and opinions of authors expressed herein do not necessarily state or reflect those of the United States Government or the University of California, and shall not be used for advertising or product endorsement purposes. 


\title{
TOWARD A SURFACE PHOTOELECTRON DIFFRACTOMETER: A PROGRESS REPORT ON SELECTED ADVANCES AND AN ASSESSMENT
}

\author{
D. A. Shirley \\ Departments of Chemistry and Physics, Pennsylvania State University, University Park, \\ PA 16802, USA \\ Yu Zheng, Barry L. Petersen, Z. Hussain, and W. A. Huff \\ J. J. Barton \\ M.S. 2-300, Lawrence Berkeley Laboratory, 1 Cyclotron Road, Berkeley, CA 94720, USA

\section{J. Terminello} \\ IBM T. J. Watson Research Center, Yorktown Heights, N.Y. 10598, USA \\ Lawrence Livermore Laboratory, Livermore CA 94550, USA
}

\begin{abstract}
X-ray diffractometric determination of atomic structures in ordered bulk systems is highly automated and has wide application. By contrast, surface crystallography, whether based on photon or electron scattering, is still in a relatively early stage of development. A summary is given of recent selected highlights in efforts to make progress toward surface photoelectron diffractometry and holography by our Berkeley-Penn State group. It is concluded that an automated photoelectron diffractometer is practical and desirable.
\end{abstract}

\section{INTRODUCTION}

To set the stage for discussing photoelectron diffractometry, two observations should be made at the outset. First, photoelectron diffraction with variable photon energies can be very effective in determining surface structures. Second, although automated diffractometry based on this phenomenon is conceptually straightforwerd, substantial development will be needed before it will be a reality. The purpose of this paper is to help stimulate this development by projecting a vision of how a photoelectron diffractometer might be developed, then by discussing a number of observations that support the feasibility of an automated diffractometric approach.

\section{SURFACE PHOTOELECTRON DIFFRACTOMETRY: A VISION.}

Let us consider an ordered surface system, consisting of a single-crystal substrate, perhaps with a submonolayer of adsorbate.
Imagine that a highly-focussed, polarized, monochromatic beam of photon' in the $x$-ray energy range impinges upon a well-defined small spot on the surface, creating photoelectrons, and that the photoelectron energy-and therefore wavenumber, $k$-can be controlled by varying the photon energy at will. These conditions are met daily in synchrotron radiation facilities throughout the world. We note in passing that a spectrum of photoelectron energies is actually generated, with peaks corresponding to atomic core levels of the substrate and adsorbate, if any, and we now limit the discussion to a single such peak. This enables us to study a particular atomic species, and we could of course study each peak in turn, deriving information about the entire system from each.

For the selected peak, photoelectrons emerge with a particular kinetic energy, $K$, given by the Einstein equation as the photon energy iess the binding energy $\mathrm{F}_{\mathrm{B}}$ and a particular magnitude of the wave vector, $k$ : 


$$
h v=E_{B}+K=E_{B}+\frac{\hbar^{2} k^{2}}{2 M}
$$

The photoelectrons are distributed in all directions throughout the $2 \pi$ radians of solid angle above the surface plane. Because we are considering only electrons possessing the total energy allowed by the Einstein equation, their intensity pattern, pbservable-after energy analysis-on a hemispherical area detector, depicted schematically in Figure 1, will be described by the interference pattern of all unscattered (direct) and elastically scattered waves. In fact, the component of this pattern which represents interference between the direct wave and all the singlyscattered waves is essentially the electron hologram of the local atomic environment around the source atom(s), corresponding to the particular wavenumber $k$.

To describe the system further, and to visualize a photoelectron diffractometer, it is useful to define a Cartesian coordinate system in real space, with the source region at the origin, the $z$ axis normal to the surface, and the $x$ and $y$ directions taken along convenient crystalline axes. Spherical polar coordinates in real space will also be useful in some situations, especially for describing aximuthal and polar angular scans of photoelectron intensity. In addition, we may envisage a $k$-space coordinate system superimposed on, and aligned with, the realspace system. It, too, may be Cartesian or spherical polar. For a fixed photon (and photoelectron) - energy, the photoelectron propagation rectors of the angular distribution pattern (hologram) all terminate on the surface of a sphere of radius $k$ in this latter coordinate system. But this pattern will be recorded on the above hemispherical area detector in real space, since the electrons have been analyzed with suitable filters to pass only those with wavenumber $k$, and since the two coordinate systems, in real space and $k$ space, were superimposed and aligned. We shall see that this hologram can be transformed to produce a threedimensional (3D) image of the local atomic environment in real space. Alternatively, angular or polar photoelectron diffraction curves could be taken along suitable paths on the hemisphere, acquiring subsets of the total diffraction information.

Another dimension can also be explored to adrantage: the photoelectron kinetic energy for the core level under study can be varied at will by adjusting the photon energy. At each photo'n energy (and electron wave number $k$ ) a hemispherical surface of radius $\mathbf{k}$ is defined by the (analyzed) photoelectrons. The third dimension in $\mathrm{k}$-space can thus be accessed, or even filled, yielding a tremendous amount of additional information. In fact, the importance of this third coordinate-variable $k$-can be appreciated by noting that the ARPEFS (angle-resolved photoemission extended fine structure) method for determining surface atomic structure is based on varying the magnitude of $k$ for a fixed direction, and that simple surface structures can typically be determined with high accuracy using ARPEFS curves from one or two directions.

The experimental aspects of a complete photoelectron diffractometer, as described above, are straightforward, and lie within the reach of existing technology. With the advent of third generation synchrotron radiation sources, the principal missing element will finally be in place. In the remainder of this survey paper, a few quite recent advances in the understanding of photoelectron diffraction are described. In most cases, these show that photoelectron diffraction, photoelectron holography, and by extension a photoelectron diffractometric methodology are cleaner, simpler, and more readily applicable than previously expected.

\section{A FEW SELECTED FEATURES OF PHOTOELECTRON DIFFRACTION.}

Photoelectron diffraction embraces the phenomena of photoelectron angular distributions and scattering. Most of these are straightforward, but typically the aspects of these phenomena which govern photoelectron diffraction, while well known, 


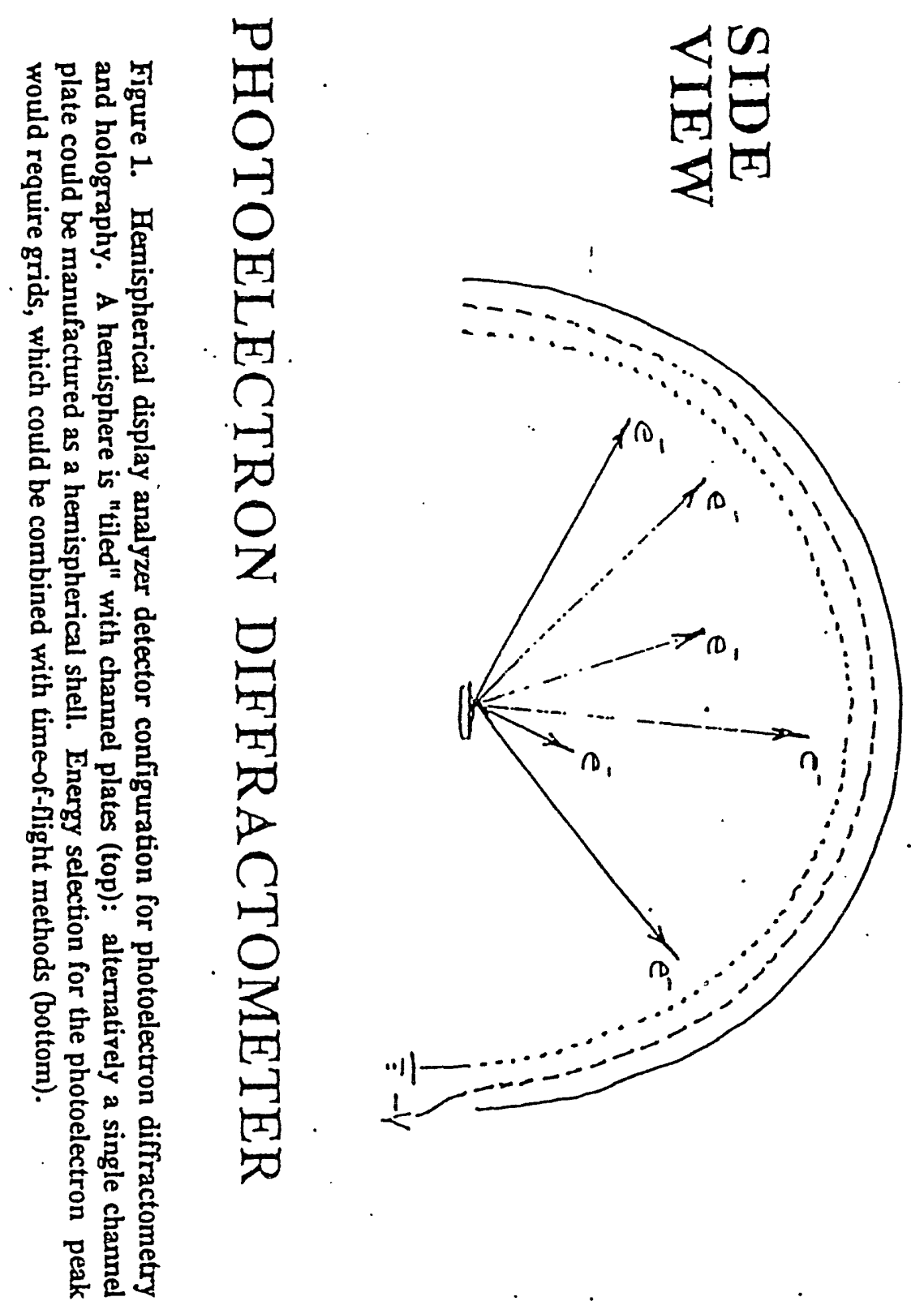

嘉

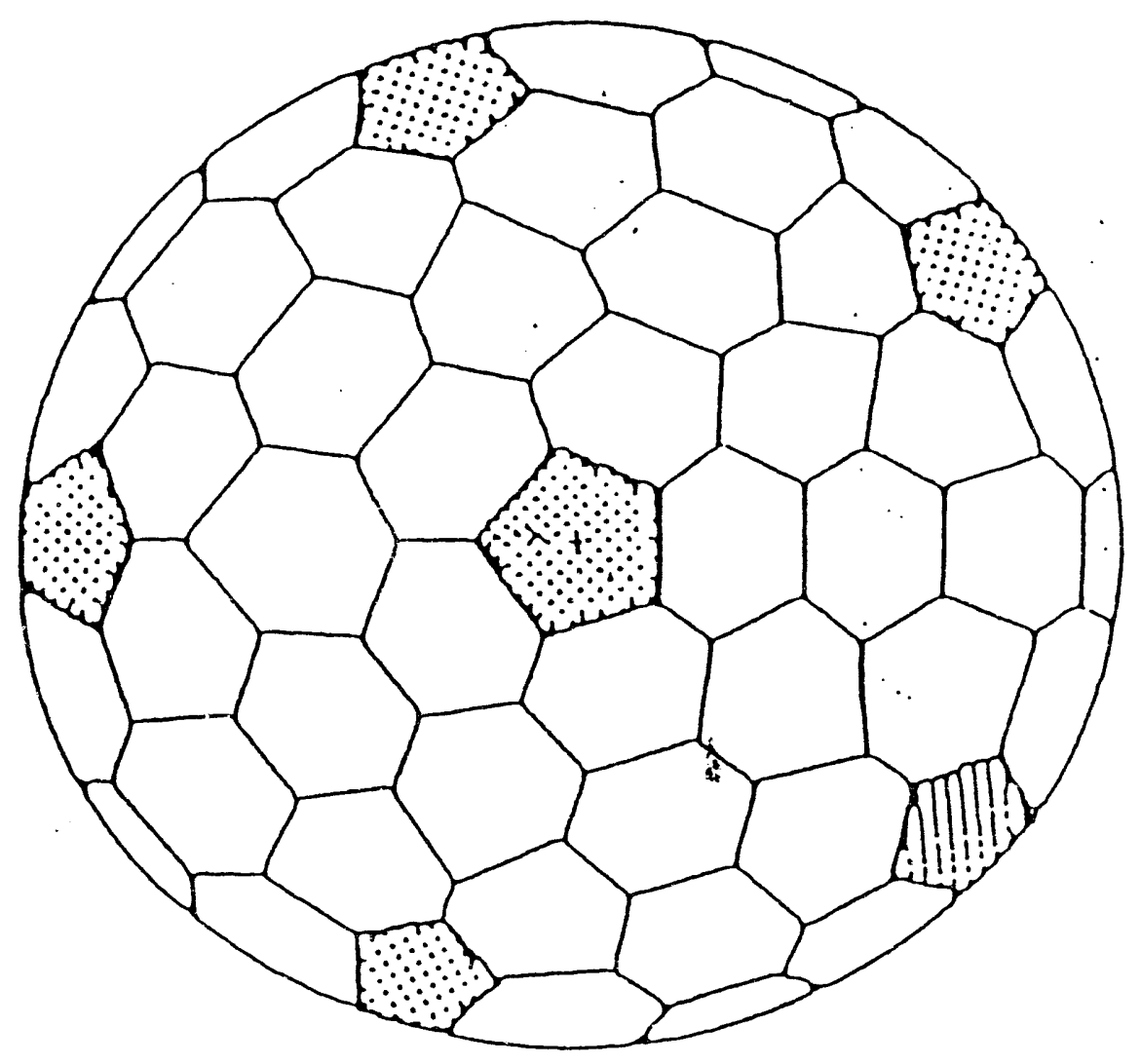


are not widely known. We discuss several such topics in turn below.

\subsection{Initial Angular Distributions and Wavefunction Phase.}

It is well-known, in photoemission from atoms, that the angular distribution of photoelectrons in the dipole limit, which covers most cases of interest to us, is given by Yang's Theorem (1),

$$
I(\theta)=1+\beta P_{2}(\cos \theta),
$$

where $\beta$ is the "asymmetry parameter" and $P_{2}(\cos \theta)$ is the second Legendre Polynomial, with $\theta$ being the angle between the electric rector and the propagation direction. An electron in an orbital of (orbital) angular momentum $l$ is emitted into $(l+1)$ and $(l-1)$ partial waves. Thus an s electron can go out only in a p-wave, with $\beta=2$ always, a $p$ electron goes into $s$ and $d$ waves, etc. At high energies the $(l+1)$ wave tends to dominate.

The above comments, which apply to closed-shell core levels of atoms, will also apply to closed shells of atoms in solids (2). The initial angular distributions of unscattered photoelectrons from the source atoms in a photoelectron diffraction experiment will therefore follow a predictable and relatively slowly-varying angular distribution function, ranging between $\sin \theta$ and $\cos \theta$, with a spherically symmetrical distribution being a distinct possibility.

The sign of the propagating wave function itself can differ toward and away from a detector placed along the electric (polarization) vector, as it will for a $p$-wave from an $s$ core level, or the sign can be the same, as for a d or $g$ wave. When the photoelectron diffraction spectrum (ARPEFS spectrum) is dominated by interference between the primary ware and singly backscattered waves, this will lead to a 180 degree phase shift in the $\chi(k)$ curves from $s$ and $p$ levels, for example (3). Conversely, observation of such a shift comprises evidence that backscattering is dominant. Shifts of this nature have been observed experimentally in this laboratory between $\chi(\mathrm{k})$ curves for photoelectrons from $\mathrm{s}$ and $\mathrm{p}$ core levels. Figure 2 shows plots of the raw $\chi(k)$ curves for the 3 s and $3 p$ peaks of $\mathrm{Cu}(111)$ taken in normal emission (4): the 180-degree phase shift is clear, especially in the higher energy range. This confirms, not surprisingly, that most of the oscillatory pattern in this ARPEFS experiment is due to backscattering: forward scattering would give the same phase in the interference pattern for the $s$ and $p$ peaks, since the interference in both cases would involve waves propagating initially toward the detector, and having the same sign for the primary and scattered wares.

\subsection{Focussing and Depth Sensitivity from Multiple Scattering}

It is well known that atoms scatter electrons elastically strongly near scattering angles of 0 and 180 degrees, and in general less strongly for angles between these two extremes. A lore has developed around this observation which facilitates ready discussion of scattering phenomena: we shall return to it and use it below: forward scattering is very strong, producing significant amounts of multiple forward scattering, and backscattering is also strong.

Multiple forward-scattering effects in atomic chains have been discussed (5-7), and the possibility of "defocussing" as well as "focussing" through multiple forward scattering in atomic chains has been discussed. Forward-focussing arises because electron waves propagating through atoms at large impact parameters are perturbed in a "focussing" sense: the electron trajectories can be regarded as being attracted toward the atomic potentials, as discussed in connection with photoelectron diffraction by Barton (8). Egelhoff has pointed out, and developed, the application of the focussing property to the study of atomic chains (9). Clearly multiple scattering is an adrantage for structural studies if it leads to focussing, because the electron intensity will be increased along directions in which atoms lie. 

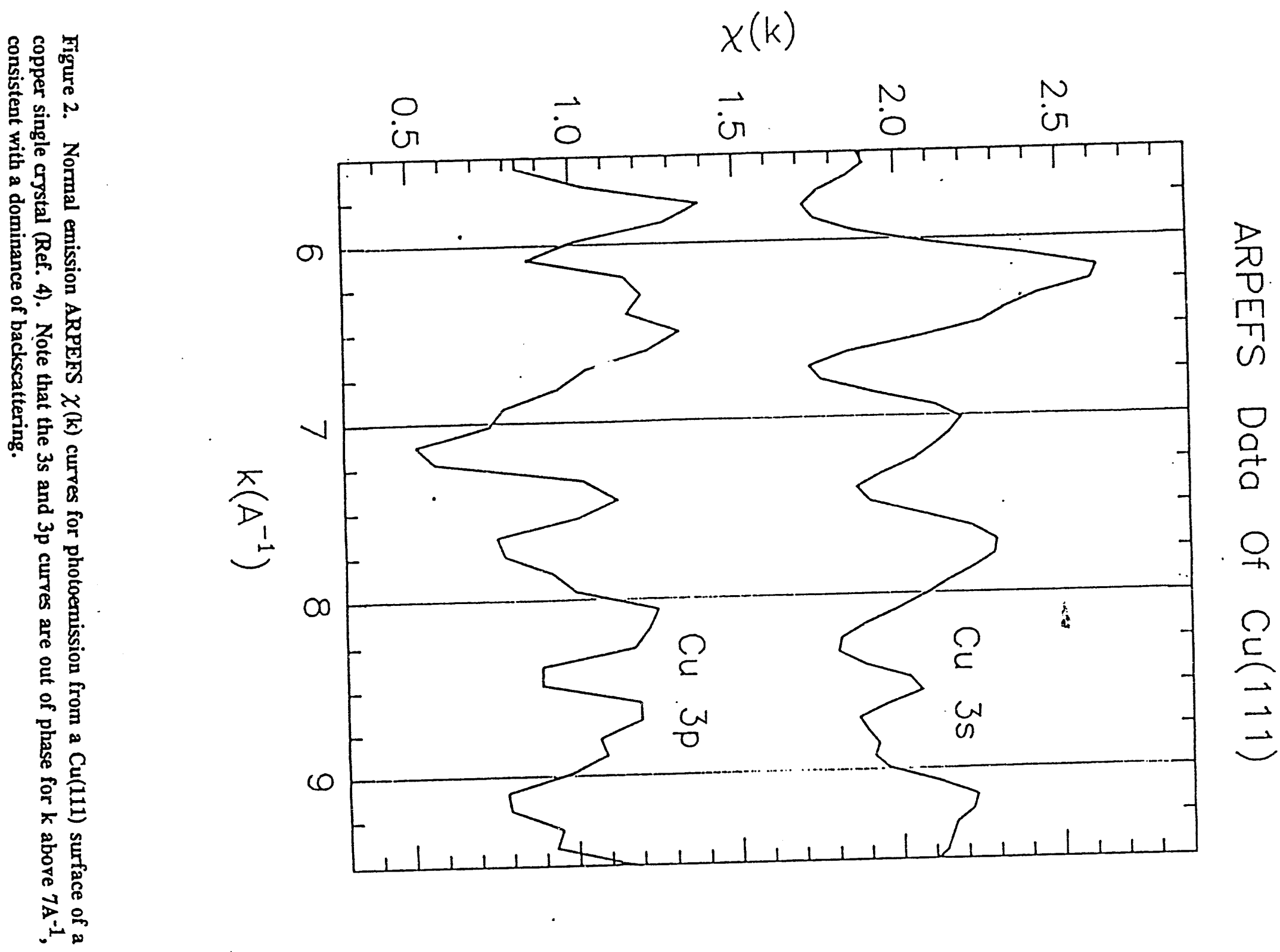
However, studies of multiple-scattering effects in atomic chains (5-7) have led to the observation that, when focussing becomes very strong, multiple scattering can actually defocus the electron "beam" as it propagates along the chain. The oversimplified interpretation that such defocussing will always occur in real systems, and that it applies to ARPEFS (the oscillatory term) as well as to forward scattering at a fixed kinetic energy, has cast doubt on the depth sensitivity of ARPEFS, the argument being that if a chain of three or more atoms defocusses the electron wave, depth sensitivities beyond two layers or so from the surface cannot be expected.

Greater depth sensitivities have actually been observed by ARPEFS for several systems, and a careful analysis on a model system (10) shows why. Backscattering dominates in ARPEFS (through the oscillatory term), and double scattering dominates among the multiple scattering channels. Small forward scattering amplitudes and phase shifts lead to convergence and constructive interference between the single- and double-scattered electron waves. Thus, for ARPEFS, multiple scattering actually enhances forward focussing and therefore the depth sensitivity, as shown in Figure 3 (10).

\subsection{Autoregression and Fourier Transforms in ARPEFS.}

The dominance of backscattering confers upon the angle-resolved photoemission extended fine structure method certain particular advantages, one of which-the extended depth sensitivity due to multiple scattering-was described above. Another advantage is the ability to measure interatomic distances quite directly and very accurately, or at least very precisely. This advantage can be attributed to two features of ARPEFS spectra, and it is perhaps best understood by comparing "energy sweeps" of photoelectron diffraction signals (i.e., ARPEFS signals) in a fixed direction in realand $k$-space, on the one hand, with aximuthal or polar sweeps at fixed photoelectron energy on the other. This comparison is best done while keeping Nyquist's Theorem in mind, although a detailed mathematical discussion will not be given here.

Naively either an azimuthal or a polarangle scan might be expected to yield an equivalent amount of information to an ARPEFS stan, because each varies one of the three spherical polar coordinates in k-space. In fact most of the angular-scan experiments reported to date have employed relatively high-energy photoelectrons, thereby emphasizing forward scattering, so meaningful comparisons are hard to make. One vary practical (and in a sense fortuitous) advantage of ARPEFS spectra is that they are collected in an optimal energy range: angular scans at similar low energies (ca. 300 eV) are also relatively information-rich. At any rate, comparison of the large body of data in the literature shows that ARPEFS spectra are typically used to determine structures, and interatomic distances in particular. The reason for this distance sensitivity can be attributed to the fact that $k$ and $\mathbf{r}$ are conjugate rariables, and distances are readily accessible from $\chi(\mathrm{k})$ curves by Fourier transform methods, although the final values for interatomic distances are derived from more quantitative curve-fitting. Distances from angle-swept spectra are more problematical: directions and symmetry properties follow more readily from these spectra, especially if they are collected at low angular resolution. We note, however, that if both angles-polar and azimuthal-are scanned, distances can be derived through holographic analysis, which can be regarded as two-dimensional Fourier inversion to yield a three-dimensional real-space image, with the length scale being in effect derived from the known length of the $k$ vector. Again it is important to work in an optimal energy range, as discussed below in the holography section.

Nyquist's Theorem can be employed, in a somewhat oversimplified way, to infer the limits of accuracy attainable from photoelectron diffraction data (11). In essence the sampling frequency in $k$-space, or 


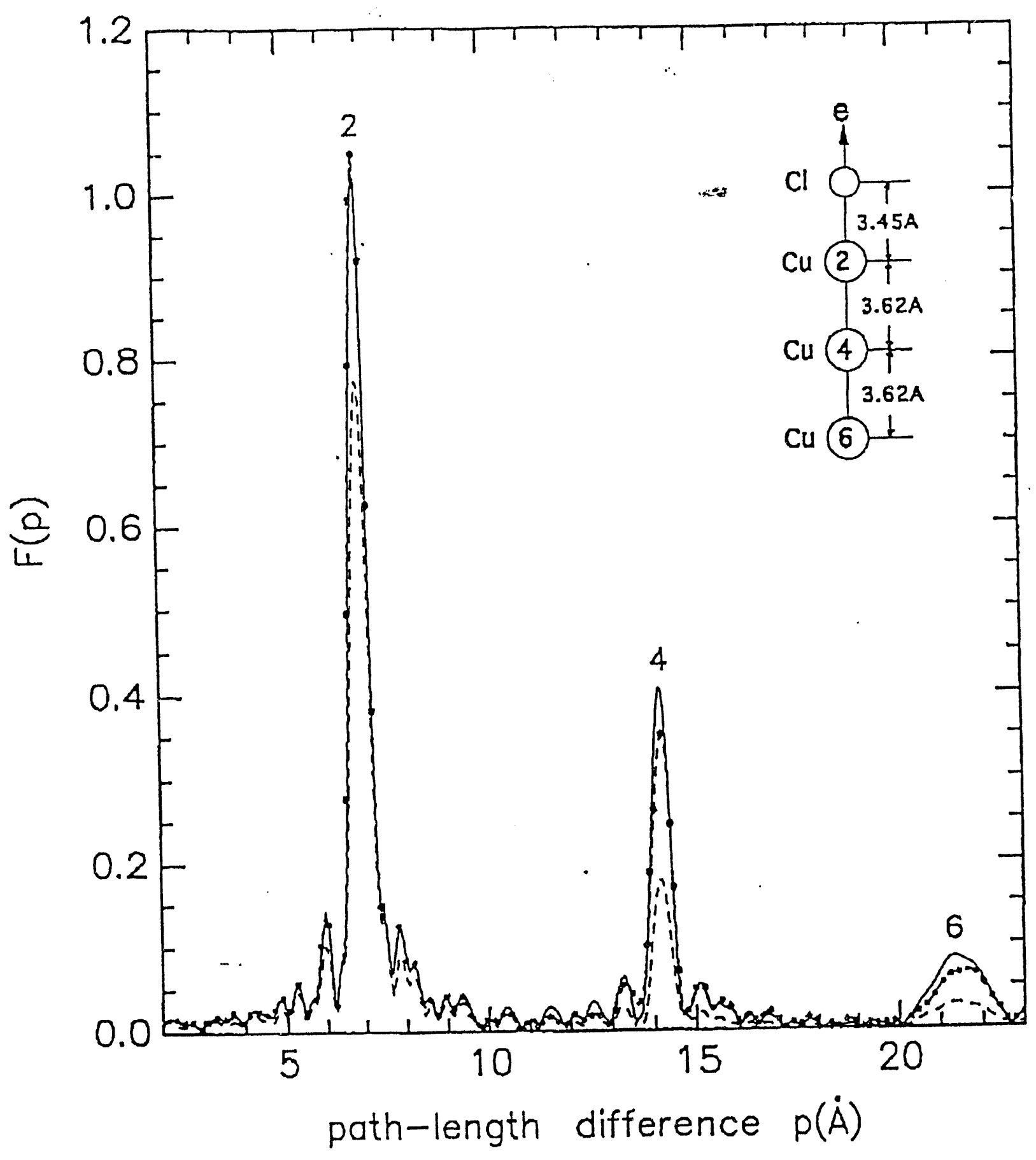

Figure 3. Model linear chain for the $\mathrm{Cl} / \mathrm{Cu}$ system, illustrating the enhancement of depth sensitivity by multiple scattering,-after Ref. 10. Dashed curve: single scattering only; filled circles: double scattering; solide curre: tenth order scattering. The increased intensity of the peak near 21 Angstroms (sixth layer) relative to that near 7 Angstroms (second layer) is due to multiple scattering. 
in angle for angle-swept spectra, should be high enough to record the harmonic content of the data. Higher data density does not contain additional information. The ARPEFS $\chi$ curves may show up to 20 maxima and minima (10 of each), and considering their spectral content, perhaps ca. 40-50 points in $k$-space are needed to carry all the information available. Application of this approach to angle-swept data supports the need for high angular resolution and energy optimization if detailed structural information is to be obtained.

Fourier transformation of an ARPEFS $\chi(\mathrm{k})$ curve (12) yields a very valuable realspace path-length difference (PLD) spectrum. To obtain optimum information, Barton showed (13) that maximum-entropy methods yield an evident resolution in the PLD spectrum that is consistent with the information content of the data. A variantauto-regressive linear prediction (ARLP)-has been used extensively by this group. Figure 4 shows a comparison of Fourier-transform spectra computed by Li-Qiong Wang for a $\mathrm{Cl} / \mathrm{Cu}$ system, with and without ARLP. Clearly the same spectral features are present in the two curres: they are just better resolved in the ARLP curve. More recently Yu Zheng in this group has improved the envelope function applied to the $\chi(\mathrm{k})$ curve before the transform, to use a function that is flat throughout most of the $k$ range, thereby preserving resolution better while suppressing sidebands effectively with curved ends.

In summary, Fourier transformation of the $\chi(\mathrm{k})$ curve yields a PLD spectrum which contains ample distance information.

\subsection{Explicit Determination of Atomic} Structure from Fourier-transform ARPEFS.

The PLD spectrum obtained as described above by Fourier-transforming the $\chi(\mathrm{k})$ curve can be used directly to determine the local atomic structure around the photoemitter without further data analysis. In fact the PLD spectrum can be superimposed on a drawing of the lattice and the peaks will fall at atomic positions. This has been confirmed by analysis of ARPEFS data on a number of systems, and it works in every case. A detailed report is in preparation (14). To illustrate this result, Figure 5 shows a comparison of an ARLP-FT ARPEFS spectrum computed by A. Schach von Wittenau for $\mathrm{S} / \mathrm{Cu}(001)$ with expected positions based on the lattice structure. Of the eight "peaks, only the one near 9 Angstroms is missed slightly, and in fact this is a consequence of lattice reconstruction in the second layer.

The implications of this result are profound. First, it should be noted that phase-shift effects are present, as in EXAFS, but that they are smaller, mainly because source-atom phase shifts cancel out in the ARPEFS effect. Because phase shifts are always present, final fitting of the $\chi(\mathrm{k})$ curves with a complete theory is desirable, but analysis (14) shows that peak positions can be determined to an accuracy of 0.3 Angstroms and structures to 0.1 Angstroms directly by this method. Even better results can be expected by triangulation, using ARLP-FT ARPEFS spectra taken in several directions. In the limit of collecting ARPEFS data over (most of) the entire angular range of the hemisphere $\theta=0-\pi, \Phi=0-2 \pi$, a "complete" photoelectron diffraction experiment, we would have a photoelectron diffractometric method for determining surface structure automatically. The simplicity and directness of the photoelectron diffraction method, free from major phase shift and multiple-scattering problems, would lend this method to automation and hence to a diffractometer for the essential structure determination. Of course final refinements of structural parameters could still be made by the use of sophisticated scattering theory.

We believe that at this point the feasibility of a photoelectron diffractometer has been demonstrated.

\subsection{Practical Photoelectron Holography.}

After Szoke (15) and Barton (16) suggested photoelectron holography, several groups have contributed to this new field. While the situation is improving, the results to date 


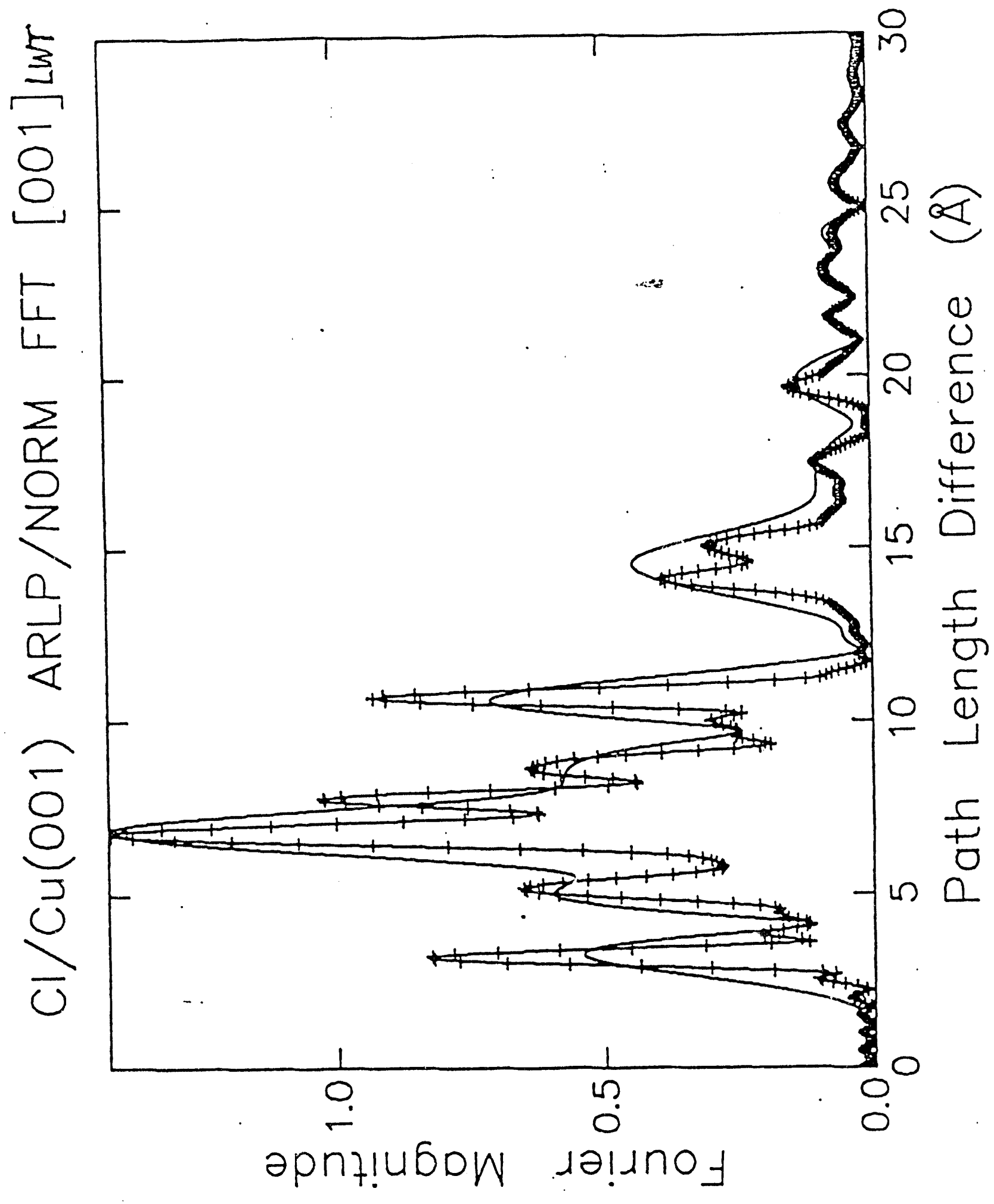

Figure 4. Comparison of the Fourier transforms for the system $\mathrm{Cl} / \mathrm{Cu}(001)-[001]$, with (crossed curve) and without (smooth curve) autoregressive linear prediction treatment. Note that all of the peaks in the ARLP curve are also present, though less well resolved, in the normal transform. 


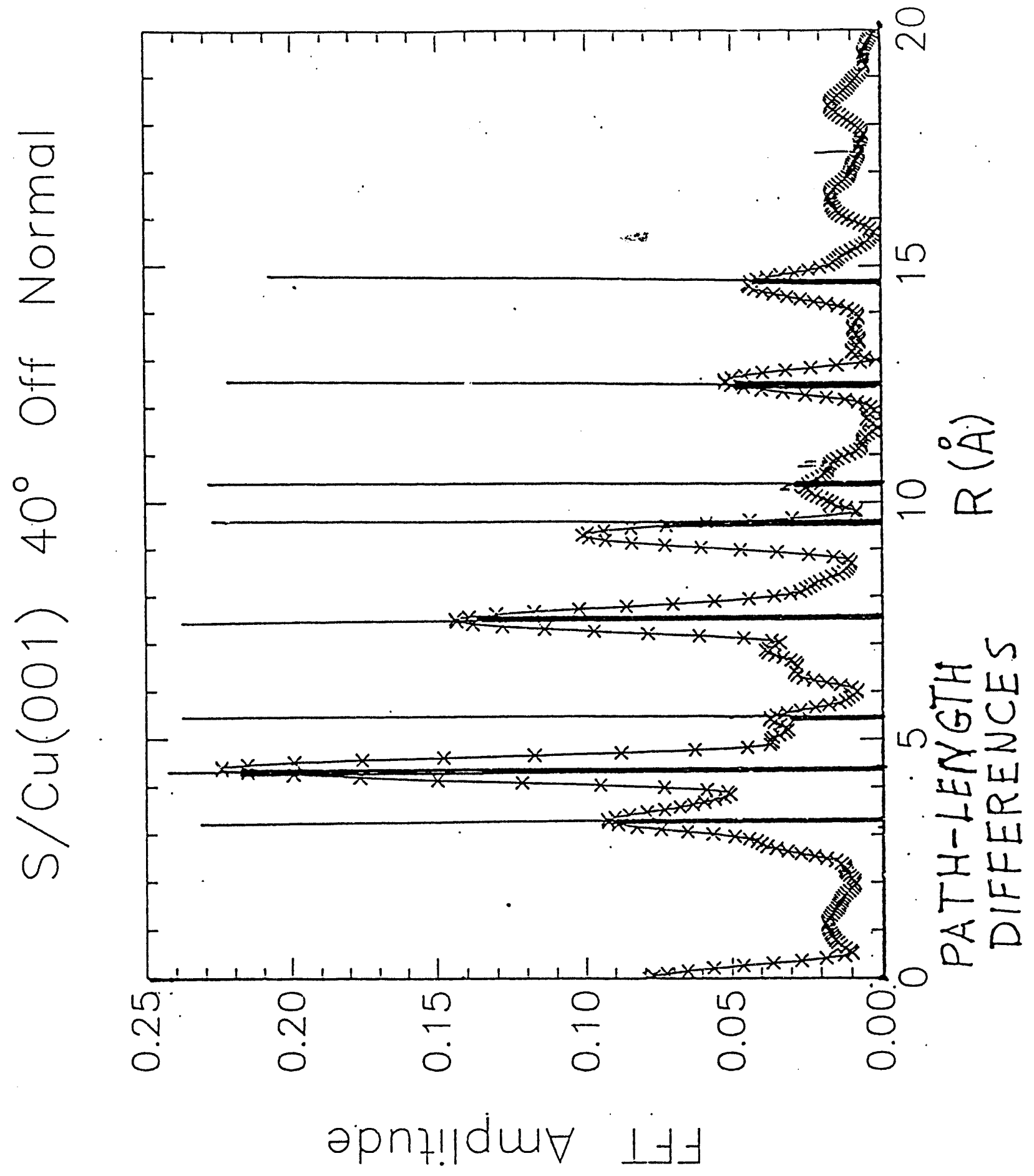

Figure 5. Comparison of an ARLP-FT ARPEFS spectrum computed by A. Schach von Wittenau, based on data reported in Ref. 11 , with peak positions computed from the lattice structure. Agreement is excellent except for peak near $9 \mathrm{~A}$, which is shifted because of lattice reconstruction. 
have been somewhat disappointing. Distorted real-space images have been reported, and phase shifts have been noted as one of sereral limitations on the method. Conclusions about the practicability of the photoelectron holography have been ambiguous and even controversial.

Petersen, et al. (17) have imaged the Pt lattice using photoelectron holography, taking advantage of two special conditions: $\varepsilon$ lange solid angle detector, and a carefully chosen intermediate energy range. The results are very encouraging. Lattice positions are registered in the real-space image to within about 5\% (ca 0.1 Angstrom) of their known positions, in the $x-y$ (transverse) direction. This is made possible by the large angular range (84 degrees) corresponding to the $x$ and $y$ directions. In the normal (z) direction, on the other hand, elongated images were obtained because only a very small range of the $k_{2}$ coordinate is permitted, given the flatness of the "polar cap" spanned by the acceptance angle of the detector. Figure 6, which is Figure 3a in reference 17, illustrates the state of the art provided by this approach. Thirteen atoms in addition to the source atom are depicted in the real-space image, which includes a total of 21 real and "twin" atomic images. The atomic images are distorted into "cigar" shapes, elongated in the $z$ direction as described above. They are also displaced along $z$, presumably due in part to phase shifts. The lattice is well represented, however, confirming the power and promise of the method. The twin-image problem is easily solved by multiple-energy experiments (18). The elongation along $z$ is well understood as a diffraction-limitation effect. It is improved by the multiple-energy approach, which provides more sampling along the $z$ direction in $k$-space, but will be more effectively ameliorated by using larger solid-angle detectors. It remains to be seen to what extent the displacement of the atomic images along $z$ will still be problematical when these two improvements have been made.

The display analyzer used in an advanced photoelectron-holography experiment is the same instrument at the front end as the proposed photoelectron diffractometer, as both approaches access all three directions in k-space. Thus diffractometry and holography can be performed on the same instrument, and they may be regarded as two methods for analyzing the same complete data set.

\section{SUMMARY}

In this paper we have presented evidence that the technology exists, and the requisite phenomena have been explored, to establish photoelectron diffractometry as a straightforward automated method for determining surface structures in ordered systems. With the new third generation of synchrotron radiation sources now coming on line, there are opportunities for developing versatile and powerful photoelectron diffractometers.

\section{ACKNOWLEDGEMENTS}

This work was supported by the Director, Office of Energy Research, Office of Basic Energy Sciences, Chemical Sciences Division of the U.S. Department of Energy, LBL under Contract No. DE-AC03-76SF00098 and LLNL Lnder Contract No. W-7405ENG-48, and by The Pennsylvania State University. The experiments were conducted at the National Synchrotron Light Source, Brookhaven National Laboratory, and at the Stanford Synchrotron Radiation Laboratory. 


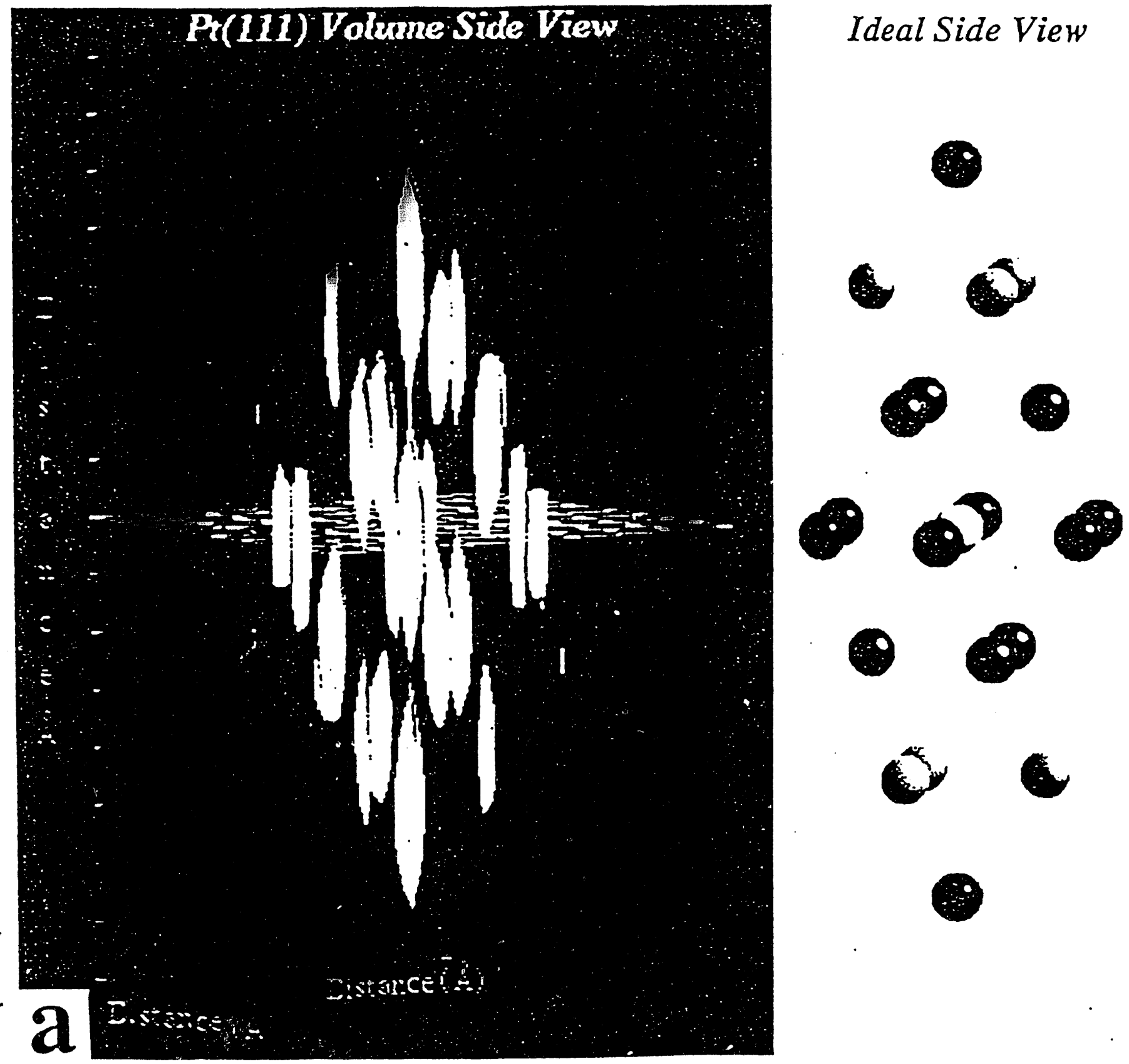

Figure 6. Three-dimensional volume side views of the Pt (111) lattice, from photoelectron holographic measurements at $351 \mathrm{eV}$ electron kinetic energy with Pt $4 \mathrm{f}$ electron peak, after Ref. 17. Thirteen atoms above the source atom are imaged, together with their "twin" images. The images are cigar-shaped because of the short sampling range in the $k_{z}$ direction in $k$ space. 


\section{REFERENCES}

1. C. N. Yang, Phys. Rev. 74, 764 (1948).

2. R. F. Daris, S. D. Kevan, B.- C. Lu, J. G. Tobin, and D. A. Shirley, Chem. Phys. Lett. 71, 448 (1980).

3. This was originally pointed out to DAS by S. Y. Tong, and was published by S. Y. Tong and J. C. Tang, Phys. Rev. B25, 6526 (1982). It was confirmed by this group for the S/Ni system, but has not been thoroughly discussed and published.

4. W. A. Huff, et al., to be published.

5. S. Y. Tong, H. C. Poon, and D. R. Snider, Phys. Rev. B32, 2096 (1985).

6. M. L. Xu, J. J. Barton, and M. A. Van Hove, Phys. Rev. B39, 8275 (1989).

7. A. P. Kaduwela, D. J. Friedman, and C. S. Fadley, J. Electron. Spectry. Relat. Phenom. 57, 223 (1991).

8. J. J. Barton and D. A. Shirley, Phys. Rev. B32, 1906 (1985). See especially Figure 3.

9. W. F. Egelhoff, CRC Critical Review in Solid State and Materials Sciences 16, 213 (1990).
10. Y. Zheng and D. A. Shirley, Chem. Phys. Lett. 203, 114 (1993).

11. Alexis Schach von Wittenau, $Z$. Hussain, L. Q. Wang, Z. Q. Huang, Z. G. Ji, and D. A. Shirley, Phys. Revi: B45, 13614 (1992).

12. Z. Hussain, D. A. Shirley, C. H. Li, and S. Y. Tong, Proc. Natl. Acad. Sci. USA 78, 5293 (1981).

13. J. J. Barton, Ph. D. Thesis, University of California, Berkeley, 1985.

14. Y. Zheng and D. A. Shirley, in preparation.

15. A. Szoke, in Short Wavelength Coherent Radiation: Generation and Applications, edited by D. T. Atwood and J. Bokor (AIP Conf. Proc. 146, New York, 1986).

16. J. J. Barton, Phys. Rev. Lett. 61, 1356 (1988).

17. Barry L. Petersen, L. J. Terminello, J. J. Barton, and D. A. Shirley, Chem. Phys. Lett., to be published.

18. Barry L Petersen, L. J. Terminello, J. J. Barton, and D. A. Shirley, in preparation. 
11
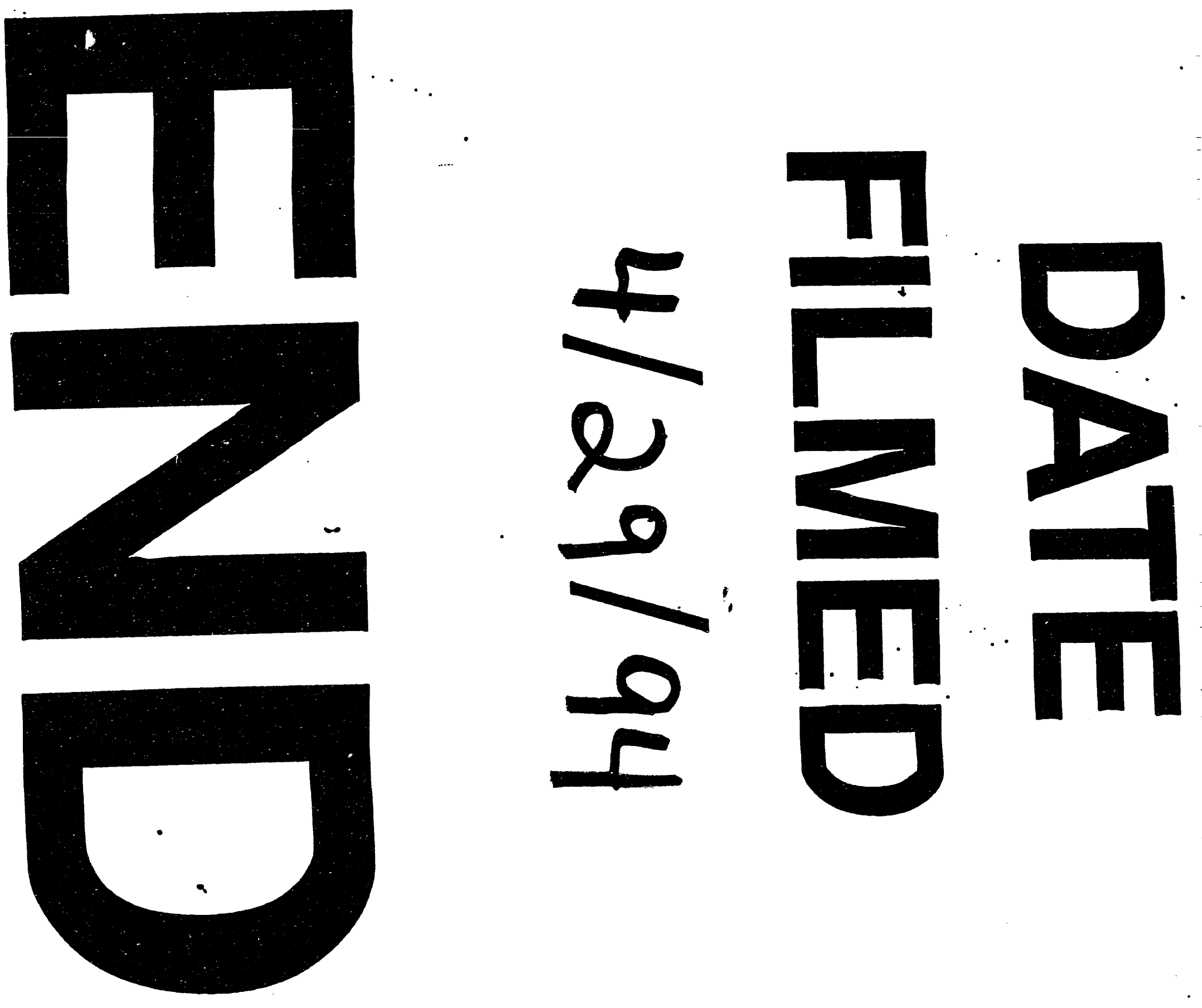


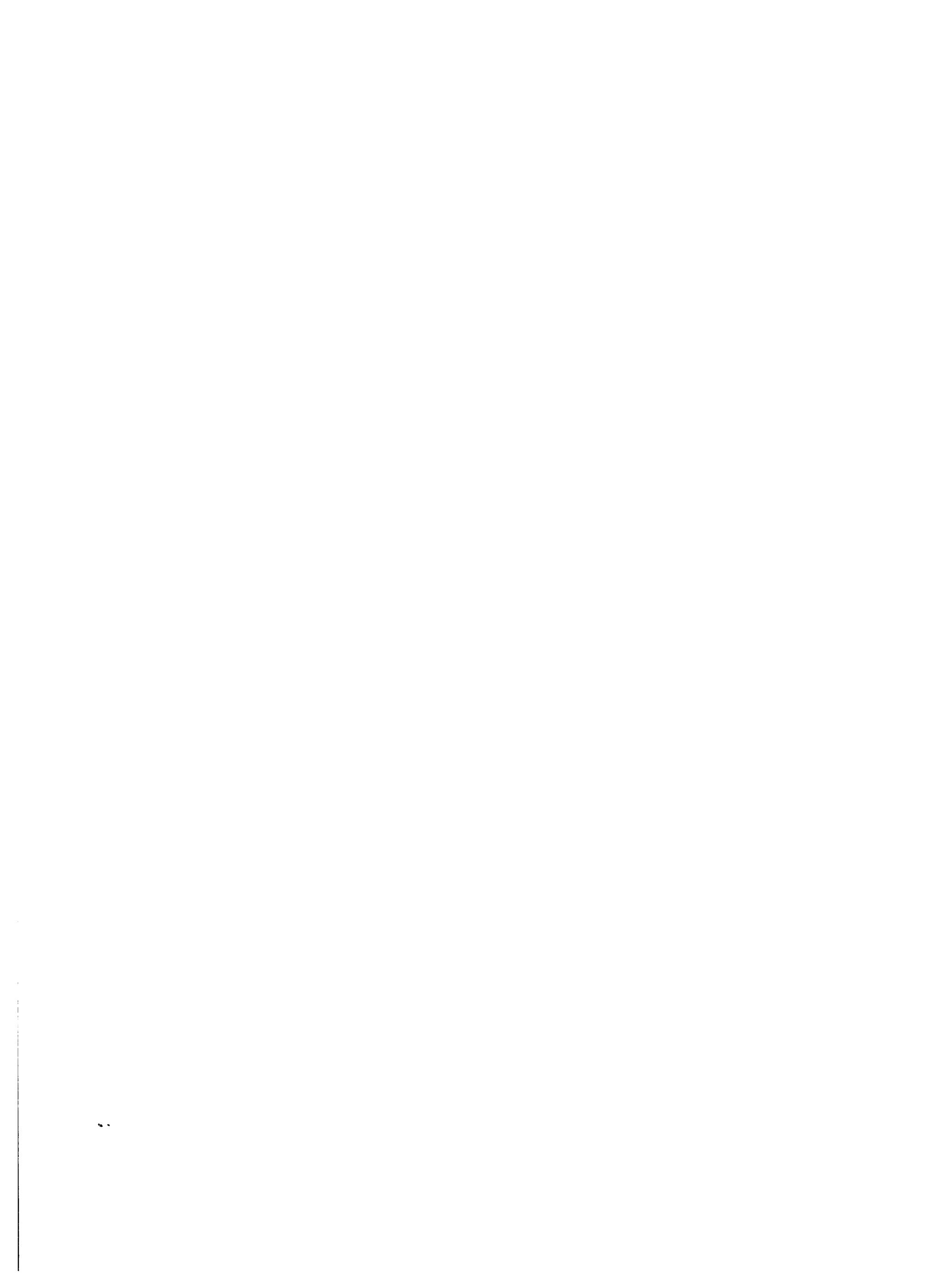

